\title{
Estado del arte del gusto graso
}

DOI: 10.17533/udea.penh.v22n1a07

PERSPECTIVAS EN NUTRICIÓN HUMANA

ISSN 0124-4108

Escuela de Nutrición y Dietética, Universidad de Antioquia. Medellín, Colombia

Vol. 22, N. ${ }^{\circ}$ 1, enero-junio de 2020, pp. 89-98.

\section{Norma Constanza López Ortiz ${ }^{1}$}

\section{Resumen}

Antecedentes: existe evidencia científica sobre la detección y reconocimiento del sabor a grasa en las papilas gustativas, y sobre la relación entre las propiedades sensoriales de los lípidos en los alimentos, la nutrición y la salud pública. Objetivo: presentar los avances investigativos en la cualidad del sabor a grasa y las estrategias actuales para lograr el cumplimiento de las recomendaciones del consumo de lípidos. Resultados: existen bases fisiológicas para afirmar que el sabor graso constituye uno de los gustos básicos, en los que están identificados sus posibles receptores y polimorfismos. La sensibilidad de estos receptores a los ácidos grasos se afecta por el consumo de grasa. La grasa dietaria se puede reducir cambiando los métodos de cocción y en la industria alimentaria usando reemplazantes de grasa. Conclusión: el gusto graso podría estar modulado por factores genéticos y ambientales. Existen variantes genéticas de los receptores y su sensibilidad depende de la grasa dietaria. Los reemplazantes de grasa son una alternativa para reducir su aporte alimentario.

Palabras clave: gusto, sustitutos de grasa, grasa alimentaria, percepción del gusto, grasa, lípidos.

1 Autor de correspondencia. Profesora asociada Universidad Nacional de Colombia sede Bogotá. MSc en Ciencias-Química Universidad Nacional de Colombia. Orcid: https://orcid.org/0000-0002-2510-3685. Correo electrónico: nclopezo @unal.edu.co

Cómo citar este artículo: López Ortiz NC. Estado del arte del gusto graso. Perspect Nutr Humana. 2020;22:89-98. DOI: 10.17533/udea.penh.v22n1a07 


\section{State of the Art of "Fat Flavor"}

\section{Abstract}

Background: Scientific evidence exists on the detection and recognition of the 'fatty taste' as one of the tastes sensed by taste buds, and around the sensory properties of fats in foods as related to nutrition and public health. Objective: Present research advances in the quality of the fatty flavor and current strategies to achieve compliance with the recommendations for lipid consumption. Results: There is physiological basis for affirming that the fatty taste constitutes one of the basic tastes, in which its possible receptors and polymorphisms are identified. Taste sensitivity to fatty acids is affected by the consumption of lipids. Dietary fat consumption can be reduced by changing cooking methods and in the food industry by using fat replacers. Conclusion: The fatty taste could be modulated by genetic and environmental factors. Genetic variants exist in taste receptors and their sensitivity depends on dietary fat consumption. Fat replacers are an alternative to help reduce dietary intake.

Keywords: Taste, Fat Substitutes, Dietary fat, Taste Perception, Fat, Lipids.

\section{INTRODUCCIÓN}

La grasa se percibe como una sensación gustativa. Este hallazgo tiene varias implicaciones que han despertado el interés de las autoridades en salud y en la industria de alimentos, debido a que los lípidos dietarios tienen una función fisiológica, nutricional y propiedades tecnológicas especiales. La recomendación de la Organización Mundial de la Salud (OMS) de reducir el consumo total de grasa a menos del $30 \%$ de la ingesta calórica diaria, para evitar un aumento malsano de peso (1), es un parámetro importante para tener en cuenta en la composición nutricional de las raciones alimenticias. La presencia de grasa como propiedad sensorial percibida por los sentidos de la vista $(2,3)$, tacto $(4,5)$ y olfato $(6,7)$ ha sido bien documentada. Mediante pruebas psicológicas se ha observado que el hambre promueve la detección rápida de imágenes de alimentos ricos en grasa, lo que parece ser razonable basados en el hecho de que la grasa permite una ingesta eficiente de energía, debido a su alta densidad energética (8).
La cualidad gustativa de la grasa es ahora estudiada como parte de los gustos básicos que incluyen el dulce, el ácido, el salado, el amargo y el umami. Algunos ácidos grasos libres son detectables a través de mecanismos de sabor en la vía oral humana, por lo que es pertinente conocer los avances en las investigaciones acerca de lo que podría ser considerado el sexto sabor, el sabor "a grasa", y cómo esta sensación se podría relacionar en algunas poblaciones con el consumo de dietas altas en calorías provenientes de los lípidos.

Se realizó una revisión bibliográfica cuyo objetivo fue documentar las evidencias científicas que aseguran que el sabor a grasa se detecta y se reconoce mediante receptores gustativos; además, identificar las posibles reformulaciones en los alimentos que cumplan con los requerimientos actuales de grasa dietética.

\section{MATERIALES Y MÉTODOS}

La consulta bibliográfica se realizó mediante el acceso a las bases de datos SciELO, Science Direct 
y PubMed. La búsqueda de información cubre la temática de preferencia de los últimos diez años. Sin embargo, se tuvieron en cuenta referencias anteriores que siguen vigentes, especialmente relacionadas con la reglamentación. Se consultaron libros de química de enseñanza universitaria.

\section{RESULTADOS Y DISCUSIÓN}

\section{El sabor de los ácidos grasos libres}

Hasta hace poco, el gusto a grasa no era reconocido como una modalidad de los sabores básicos. Sin embargo, la creciente evidencia de estudios en humanos y animales indica la existencia de una modalidad de sabor que responde a la presencia de grasa en la boca debido a la identificación de receptores de ácidos grasos no esterificados en las células gustativas (9).

La percepción de un sabor comienza con el contacto con receptores gustativos, ubicados en la parte superior de las células gustativas, las cuales se hallan agrupadas en racimos de alrededor de 50-100 células que forman los llamados botones gustativos. Estos últimos se encuentran dispersos en estructuras visibles a simple vista llamadas papilas gustativas, ubicadas en superficies del epitelio de la lengua, paladar, faringe, laringe y esófago superior. Obviamente, las células receptoras del gusto a grasa se localizan en las papilas gustativas (10). Se conoce que en la percepción del sabor graso están implicados los receptores acoplados a proteínas $\mathrm{G}$ (GPCR) (11), particularmente el 120 (12) y el transportador de ácidos grasos $\mathrm{CD} 36$. Recientemente se ha demostrado que estos receptores se expresan en las papilas circunvaladas, foliadas y fungiformes localizadas en la lengua (13). Además de ser un receptor de ácidos grasos libres, el GPR120 regula varias funciones celulares, desempeña un papel crítico en el metabolismo de los lípidos (14) y se resalta que está involucrado en el control del apeti- to, la sensibilidad a la insulina y la regulación de la inflamación (15).

El descubrimiento del CD36 tiene orígenes complejos, heterogéneos y redundantes, que dan lugar a una multitud de nombres alternativos (FAT [translocasa de ácidos grasos], GPIIllb [glicoproteína plaquetaria IIIb], GPIV [receptor Bven], receptor de colágeno tipo I, receptor de trombospondina, PAS IV y CHDS 7 [susceptibilidad a la enfermedad coronaria]). La nomenclatura refleja la multifuncionalidad de la proteína y los continuos desafíos para establecer mecanismos de sus actividades biológicas (16). La interacción de los ácidos grasos con esos receptores desencadena la secreción de $\mathrm{Ca}^{2+}$ intracelular, la cual luego induce la liberación de neurotransmisores que incluyen noradrenalina, serotonina (5-hidroxitriptamina, 5-HT), aminoácidos (glutamato y ácido $\mathrm{y}$-aminobutírico) y acetilcolina (Ach), de los receptores del gusto oral y de las hormonas intestinales, incluida la colecistoquinina (CCK), el péptido YY (PYY), el péptido 1 similar al glucagón (GLP-1) y el péptido liberador de insulina dependiente de la glucosa (GIP), de las células enteroendocrinas, que luego interactúan con receptores en vías aferentes parasimpáticas para transmitir la información al cerebro (17).

Las investigaciones sobre el gusto graso son consistentes sobre la participación de la lipasa lingual y en que los estímulos detectados son los ácidos grasos libres (AGL) y no los triglicéridos (18). Determinar la actividad lipolítica lipasa lingual y su papel fisiológico es importante para establecer su incidencia en la percepción del gusto de los ácidos grasos (19). Los receptores del gusto podrían adaptarse a la concentración de compuestos de sabor endógenos en la saliva; además, se propone que puede existir un nivel de fondo de ácidos grasos, así como el catión sodio en el caso del sabor salado y que la sensibilidad al sabor de los ácidos grasos podría 
ser modulada por la concentración de AGL en la saliva (20).

Los cuatro ácidos grasos más abundantes en la saliva son el palmítico (C16:0), el esteárico (C18:0), el oleico (C18:1n9) y el linoleico (C18:2 n6) (20-22). El ácido oleico ha sido usado como referencia en estudios de determinación de umbrales de detección del sabor graso. Los umbrales de detección pueden variar, debido a que la percepción de la grasa puede ser influenciada por factores genéticos, neuronales y personales, como la dieta y la sensibilidad para el componente amargo 6-n-propiltiouracilo (PROP); aunque los resultados en los factores genéticos no han sido suficientes para sacar conclusiones definitivas (23). Se encontró correlación positiva entre el índice de masa corporal (IMC) y los umbrales de detección de los sabores graso y amargo en población obesa y sana con polimorfismo genético de un solo nucleótido (SNP) de los receptores CD36 (rs1761667) y TAS2R38 (rs1726866 y rs10246939), aunque aún no hay resultados definitivos sobre cómo afectan estos polimorfismos la detección del gusto graso y su influencia sobre la ingestión calórica y el IMC.

Los resultados de estudios con participantes obesos mostraron una correlación positiva entre los umbrales de detección del ácido linoleico y el 6-npropiltiouracilo (24). Se ha estudiado la asociación entre el IMC, la ingesta dietética y la percepción de la grasa oral y, aunque los resultados no son contundentes, no se ha encontrado una relación significativa entre el IMC y los umbrales de detección de ácido oleico, aceite de parafina, aceite de canola, ni con el aceite de canola enriquecido con ácido oleico (25), pero sí se ha demostrado que el aumento del contenido de grasa en la dieta ocasiona un incremento en la preferencia por los alimentos altos en grasa; esto se ha relacionado con el IMC, ya que los umbrales de sabor a grasa parecen modificarse de acuerdo con la dieta (26).
La sensibilidad del sabor al ácido oleico está modulada por la exposición o la restricción de la grasa en la dieta durante un período de cuatro semanas. Los resultados sugieren que las diferencias en la sensibilidad del sabor a los ácidos grasos son el resultado de la adaptación gustativa a una dieta alta en grasas y pueden contribuir al exceso de grasa, debido a una respuesta de sabor atenuada a los ácidos grasos entre las personas que habitualmente consumen una dieta alta en grasas (27). De los datos presentados se deduce la importancia de los factores ambientales en cuanto a la detección del gusto graso y sus implicaciones para el desarrollo de la obesidad.

Se ha observado una amplia variabilidad en la sensibilidad al gusto de los ácidos grasos no esterificados en un mismo individuo y entre diferentes poblaciones, así como en diferentes grupos de investigación $(28,29)$. Parte de esta variabilidad puede deberse a la naturaleza hidrófoba de los AGL y de los métodos empleados para elaborar las emulsiones necesarias, para la presentación de los estímulos y posterior acceso a los receptores del gusto. Además, las propiedades de las emulsiones también pueden detectarse mediante señales somatosensoriales, que complican la atribución de los hallazgos sensoriales al gusto (30). Adicionalmente, se ha observado que si se hacen repeticiones y entrenamiento en las pruebas, ocurre el aprendizaje (rendimiento mejorado de las pruebas), cuando se usan métodos tradicionales para medir sensibilidad a los AGL; estos métodos pueden contribuir en gran medida a la variabilidad dentro del sujeto si la prueba no está estandarizada (31). Se ha propuesto que la sensibilidad oral en los humanos a los AGL aumenta al incrementar la insaturación de la cadena alquílica; sin embargo, un estudio en humanos reportó que la sensibilidad más alta fue para el ácido linoleico (C18:2n6), seguida por el a-linolénico (C18:3n3) y, finalmente, por el oleico (C18:1n9) (32). Extender la cadena alquílica de AGL crea un cambio per- 
ceptual de la acidez, la calidad experimentada en una longitud de 10 carbonos es claramente distinta (33). Los ácidos grasos de cadena media como el ácido decanoico pueden tener su propia sensación única de AGL picante 0 irritante (34). En contraste, los ácidos grasos de cadena corta o volátiles (AGV) (AGV C2- C4), como el ácido acético (C2:0), tienen notas de sabor ácido.

\section{Los lípidos en los alimentos}

Los lípidos son un grupo heterogéneo de compuestos que incluye grasas, aceites, esteroides, ceras y compuestos relacionados más por sus propiedades físicas que por sus propiedades químicas (35). La fracción no polar de los tejidos animales y vegetales se expresa como porcentaje de grasa total; sus mayores constituyentes son los triglicéridos, que representan de 93 a $95 \%$ del total, el resto corresponde a diversos compuestos que incluyen, entre otros, vitaminas liposolubles y esteroides, entre los que se encuentra el colesterol y hormonas esteroideas. El colesterol es quizá el esteroide mejor conocido, debido a su relación con la aterosclerosis y otras enfermedades. La esterificación de los triglicéridos involucra la molécula del glicerol, un triol. De esta manera, se tienen ácidos grasos esterificados y ácidos grasos no esterificados (libres) (36).

Durante los últimos años, ha recibido gran atención la interacción en los factores genéticos y ambientales sobre la percepción del gusto y sus implicaciones sobre el desarrollo de la obesidad, especialmente en los niños. En este contexto, y puesto que está descrito que el tipo de AGL en la boca influye sobre la detección del gusto graso, es importante conocer el perfil de ácidos grasos de la leche materna, por ser el primer estímulo de sabor al que están expuestos los niños recién nacidos. El perfil de los ácidos grasos de la leche materna, considerada como ideal para los bebés, contiene ácido oleico, el más abundante, y la cantidad total de poliinsatura- dos es relativamente constante durante la lactancia con un promedio de 1,3 y $17 \%$, respectivamente (37). Cabe recordar que el ácido oleico (C18:1n9) es uno de los cuatro predominantes en la saliva $y$, entre los insaturados de cadena larga, es el que menor sensibilidad al gusto graso presenta.

Los cuatro ácidos grasos saturados más abundantes en la leche materna son láurico (C12:0), mirístico (C14:0), palmítico (C16:0) y esteárico (C18:0). Los ácidos grasos saturados de cadena impar son raros en la naturaleza, y en la dieta humana provienen principalmente de las grasas lácteas (38). Los niveles elevados de ácido palmítico (C16:0) en la leche materna pueden estar potencialmente relacionados con el consumo de aceite de palma. Gracias a la revisión de otros estudios, se podría concluir que los aceites, la carne y el consumo de pescado están fuertemente reflejados en la composición de ácidos grasos de la leche humana y que la dieta confiere una huella digital clara y distinguible en la grasa de este fluido biológico (39). También se ha propuesto que la estimulación del gusto graso por los AGL saturados es menor que en los polinsaturados, como se mencionó anteriormente (32).

Las entidades de salud aconsejan limitar el consumo de grasas saturadas a menos del $10 \%$ de la ingesta calórica diaria; también limitar el consumo de grasas trans a menos del $1 \% \mathrm{y}$, además, sustituir las grasas saturadas y las grasas trans por grasas no saturadas (1), en particular, por grasas poliinsaturadas. Los valores recomendados para consumo diario de calorías y su aporte de las grasas han sido bien establecidos y varían dependiendo de diferentes factores como la edad, el sexo, el peso, la altura y el nivel de actividad física (40).

\section{Los reemplazantes de grasa}

Se han abordado varias estrategias para disminuir los factores de riesgo de enfermedades crónicas 
ocasionadas por la elección de la dieta, especialmente en la reformulación de alimentos. Ha sido un gran desafío reducir la grasa de los alimentos conservando su sabor y textura. Existen diferentes maneras de disminuir la grasa en los alimentos, lo primero y más fácil es modificar los procesos de cocción; por ejemplo, cambiar la fritura por horneado o cocción a la plancha. La utilización de ingredientes con un menor contenido de grasa, por ejemplo, la utilización de leche descremada y el uso de sustitutos de grasa. La investigación en la reformulación de alimentos está relacionada con los problemas tecnológicos en su procesamiento, la funcionalidad, los atributos sensoriales, la conservación de alimentos o la obtención de reemplazos nuevos y más saludables de sal, grasa, azúcar (41). Así, se logra desarrollar alimentos nutricionalmente optimizados (42). Con el conocimiento actual de las características físicas, químicas y sensoriales de los lípidos, se ha logrado reemplazar el contenido de grasa en los alimentos industrializados por otro tipo de sustancias, sustitutos, miméticos, reemplazantes 0 análogos de grasa, porque la sensación de grasa depende principalmente del tamaño y la reología de las partículas o las gotas, y mucho menos de la naturaleza química del ingrediente (43).

Los biopolímeros se pueden utilizar para modular las características texturales y sensoriales de los productos reemplazantes de grasa. La mayor cantidad de biopolímeros son hidrocoloides, cuyas propiedades funcionales, como la textura, la viscosidad y la sensación en la boca, les permiten imitar las características sensoriales y de flujo de manera similar a la grasa. Las dos clases principales de biopolímeros alimentarios son las proteínas y los polisacáridos, que se pueden usar solos o en combinaciones únicas en productos alimenticios reducidos en grasa (44). Los polisacáridos incluyen gomas, pectinas, fibra soluble, almidones y maltodextrinas. Se han desarrollado diferentes métodos para modificar las macromoléculas, como la hidró- lisis mediante el uso de enzimas, para degradar el almidón y reducir el tamaño de partícula. Por ejemplo, en la hidrólisis enzimática de almidón de maíz, se obtuvo una distribución del tamaño de partícula de alrededor de 2-4 um (45) para dar textura cremosa y propiedades de superficie. Los sustitutos de grasa a base de almidón han mostrado una eficacia prometedora en la sustitución de grasas y generalmente se consideran seguros. Existe evidencia muy limitada sobre cómo los sustitutos de grasa a base de almidón pueden reducir el riesgo de enfermedad cerebrovascular y obesidad en comparación con la grasa; el alcance de los efectos secundarios permanece desconocido (46).

La celulosa y sus derivados se pueden usar como sustitutos de grasa $(47,48)$. Los ésteres de ácidos grasos y un azúcar como la sacarosa, maltosa, fructosa, etc., proporcionan las características físicas de las grasas y los aceites sin ser calóricos (49). La hidrólisis enzimática, la modificación química y los tratamientos combinados con cizallamiento térmico de algunas proteínas logran asemejar las propiedades fisicoquímicas de los lípidos para uso en emulsiones semisólidas (50), alimentos como helados de crema (51), lácteos, cárnicos $(52,53)$ y productos de panadería (54). La posición de la American Dietetic Association es que la mayoría de los sustitutos de grasa, cuando se usan con moderación por adultos, pueden ser complementos seguros y útiles para reducir el contenido de grasa de los alimentos y pueden desempeñar una función en la disminución de la energía dietética total y la ingestión de grasas (55); además, posibilita continuar con el desarrollo de nuevos productos con buenas propiedades sensoriales y nutricionales. Por supuesto, es importante utilizar estas tecnologías con prudencia para no promover el consumo excesivo pasivo de alimentos. Por ejemplo, puede ser más beneficioso reducir el contenido de calorías de un producto alimenticio que ya es parte integral de una dieta (por ejemplo, una pasta para untar, aderezo 0 
salsa) en lugar de promover un mayor consumo de alimentos menos saludable (56).

En conclusión, el sabor a grasa se ha establecido como uno de los gustos básicos, se han identificado varios receptores implicados y su ubicación en las papilas gustativas. El gusto por la grasa podría estar modulado por factores genéticos y ambientales, puesto que se han identificados polimorfismos de los receptores gustativos a la grasa, aunque aún no se sabe con precisión las implicaciones de este hallazgo. Se ha podido establecer que la sensibili- dad del sabor a los ácidos grasos es el resultado de la adaptación gustativa a dietas altas en grasa, las que, se sabe, tienen efectos adversos sobre la salud. Sin embargo, el aporte de grasa de los alimentos se puede modular cambiando los métodos de cocción y, a nivel industrial, utilizando de manera apropiada los reemplazantes de grasa.

\section{CONFLICTO DE INTERESES}

La autora declara que no existen conflictos de intereses.

\section{Referencias}

1. OMS. Informe sobre la situación mundial de las enfermedades no transmisibles 2014. "Cumplimiento de las nueve metas mundiales relativas a las enfermedades no transmisibles: una responsabilidad compartida". Ginebra; 2014. [Internet]. [Citado abril de 2018]. Disponible en: http://apps.who.int/iris/bitstream/10665/149296/1/

2. Pflanzer SB, de Felício PE. Moisture and fat content, marbling level and color of boneless rib cut from Nellore steers varying in maturity and fatness. Meat Sci. 2011;87:7-11. http://doi.org/10.1016/j.meatsci.2010.08.009

3. Méndez-Cid FJ, Lorenzo JM, Martínez S, Carballo J. Oxidation of edible animal fats. Comparison of the performance of different quantification methods and of a proposed new semi-objective colour scale-based method. Food Chem. 2017; 217:743-49. http://doi.org/10.1016/j.foodchem.2016.09.009

4. Guichard E, Galindo-Cuspinera V, Feron G. Physiological mechanisms explaining human differences in fat perception and liking in food spreads-a review. Trends Food Sci Tech. 2018;74:46-55. http://doi.org/10.1016/j.tifs.2018.01.010

5. Han P, Fark T, A de Wijk R, Roudnitzky N, lannilli E, Seo HS, et al. Modulation of sensory perception of cheese attributes intensity and textura liking via ortho- and retro-nasal odors. Food Qual Prefer. 2019;73:1-7. http://doi.org/10.1016/j.foodqual.2018.11.019

6. Guichard E, Salles C, Morzel AM. Characterization of aroma compounds: Structure, physico-chemical and sensory properties. En: Guichard E, Salles C, Morzel AM, Le Bon A-M (eds.). Flavour, from food to perception. UK: Wiley Blackwell, Chichester; 2017, pp. 126-153.

7. Trautmann J, Gertheiss J, Wicke M, Mörlein D. How olfactory acuity affects the sensory assessment of boar fat: A proposal for quantification. Meat Sci. 2014;98: 255-62. http://doi.org/10.1016/j.meatsci.2014.05.037

8. Sawada R, Sato W, Minemoto K, Fushiki T. Hunger promotes the detection of high- fat food. Appetite. 2019;142:104377. http://doi.org/10.1016/j.appet.2019.104377

9. Tucker RM, Mattes RD, Running CA. Review Article Mechanisms and effects of "fat taste" in humans. Biochem Mol Biol Int. 2014;40:313-326. http://doi.org/10.1002/biof.1162

10. Besnard P, Passilly-Degrace P, Khan NA. Taste of fat: a sixth taste modality? Physiol Rev. 2016; 96:151-76. http://doi.org/10.1152/physrev.00002.2015

11. Galindo MM, Voigt N, Stein J, Van Lengerich J, Raguse JD, Hofmann T, et al. G Protein-Coupled Receptors in Human Fat Taste Perception. Chem Senses 2012;37:123-39. http://doi.org/10.1093/chemse/bjr069 


\section{El gusto a grasa}

12. Cartoni C, Yasumatsu K, Ohkuri T, Shigemura N, Yoshida R, Godinot N, et al. Taste Preference for Fatty Acids Is Mediated by GPR40 and GPR120. Journal Neurosci. 2010; 30(25):8376-82. http://doi.org/10.1523/JNEUROSCI.0496-10.2010

13. Simons PJ, Kummer JA, Luiken JJ, Boon. Apical CD36 immunolocalization in human and porcine taste buds from circumvallate and foliate papillae. Acta Histochem. 2011; 113:839-43. http://doi.org/10.1016/j.acthis.2010.08.006

14. He Q, Zhu S, Lin M, Yang Q, Wei L, Zhang J, et al. Increased GPR120 level is associated with gestational diabetes Mellitus. Biochem Bioph Res Co. 2019;512:196-201. http://doi.org/10.1016/..bbrc.2019.03.034

15. Dong-Soon I. FFA4 (GPR120) as a fatty acid sensor involved in appetite control, insulin sensitivity and inflammation regulation. Mol Aspects Med. 2018;64:92-108. http://doi.org/10.1016/..mam.2017.09.001

16. Peña-Portillo GC. Oleogustus: El Sexto Sabor en la Industria Alimentaria. Ciencia y Tecnología de alimentos. 2019; 29(3):70-5. Disponible en: https://revcitecal.iiia.edu.cu/revista/index.php/RCTA/article/view/79/67

17. Stewart JE, Feinle-Bisset $\mathrm{C}$, Keast RSJ. Fatty acid detection during food consumption and digestion: Associations with ingestive behavior and obesity. Prog Lipid Res. 2011;50(3):225-33. http://doi.org/10.1016/.jplipres.2011.02.002

18. Kawabata $Y$, Kawabata F, Nishimura S, Tabata S. Oral lipase activities and fat-taste receptors for fat-taste sensing in chickens. Bioch Bioph Res Co. 2018; 495:131-5. http://doi.org/10.1016/.bbrc.2017.10.125

19. Brignot $\mathrm{H}$, Feron $\mathrm{G}$. Oral lipolysis and its association with diet and the perception and digestion of lipids: A systematic literature review. Arch Oral Biol. 2019;108:104550. http://doi.org/10.1016/j.archoralbio.2019.104550

20. Neyraud $\mathrm{E}, \mathrm{Cabaret} \mathrm{S}$, Brignot $\mathrm{H}$. The basal free fatty acid concentration in human saliva is related to salivary lipolytic activity. Sci rep-uk.2017;7:5969. http://doi.org/10.1038/s41598-017-06418-2

21. Moon JY, Kong TY, Jang HJ, Kang HC, Cho YY, Lee JY, et al. Simultaneous quantification of 18 saturated and unsaturated fatty acids and 7 sterols as their tert-butyldimethylsilyl derivatives in human saliva using gas chromatography-tandem mass spectrometry. J Chromatogr B. 2018;1092:114-21. http://doi.org/10.1016/j.jchromb.2018.06.003

22. Kulkarni BV, Wood KV, Mattes RD. Quantitative and qualitative analyses of human salivary NEFA with gas-chromatography and mass spectrometry. Front Physiol. 2012;3:328. https://doi.org/10.3389/fphys.2012.00328

23. Shen $\mathrm{Y}, \mathrm{Kennedy} \mathrm{OB}$, Methven $\mathrm{L}$. The effect of genotypical and phenotypical variation in taste sensitivity on liking of ice cream and dietary fat intake Food Qual Prefer. 2017;55:79-90. http://doi.org/10.1016/.ffoodqual.2016.08.010

24. Karmous I, Plesník J, Khan AS, Serý O, Abid A, Mankai A, et al. Orosensory detection of bitter in fat-taster healthy and obese participants: Genetic polymorphism of CD36 and TAS2R38. Clin Nutr. 2018; 37:313-20. http://doi.org/10.1016/..clnu.2017.06.004

25. Newman LP, Torres SJ, Bolhuis DP, Keast RSJ. The influence of a high-fat meal onfat tastethresholds.Appetite.2016;101:199-204. https://doi.org/10.1016/j.appet.2016.03.011

26. Keast RSJ. Effects of sugar and fat consumption on sweet and fat taste. Curr Opin Behav Sci. 2016;9:55-60. http://doi.org/10.1016/j.cobeha.2015.12.003

27. Stewart JE, Keast RSJ. Recent fat intake modulates fat taste sensitivity in lean and overweight subjects. Int $\mathrm{J}$ Obes. 2012;36:834-42.

28. Stewart JE, Seimon RV, Otto B, Keast RSJ, Clifton PM, Feinle-Bisset C. Marked differences in gustatory and gastrointestinal sensitivity to oleic acid between lean and obese men. Am J Clin Nutr. 2011; 93:703-11. http://doi.org/10.3945/acn.110.007583

29. Running CA, Mattes RD, Tucker RM. Fat taste in humans: Sources of within- and between-subject variability. Prog Lipid Res. 2013; 52: 438-45. http://doi.org/10.1016/.jplipres.2013.04.007 
30. Teo PS. Training of a Dutch and Malaysian sensory panel to assess intensities of basic tastes and fat sensation of commonly consumed foods. Food Qual Prefer. 2018;65:49-59. http://doi.org/10.1016/j.foodqual.2017;11.011

31. Martínez NR, López JA, Wall A, Jiménez JA, Angulo 0 . Oral fat perception is related with body mass index, preference and consumption of high-fat foods. Physiol Behav. 2014;129:36-42. http://doi.org/10.1016/.jphysbeh.2014.02.010

32. Running CA, Mattes RD. Humans are more sensitive to the taste of linoleic and a-linolenic than oleic acid. Am J Physiol Gastrointest Liver Physiol. 2014;308:G442-49. http://doi.org/10.1152/ajpgi.00394.2014

33. Running CA, Craig BA, and Mattes RD. Oleogustus: The Unique Taste of Fat. Chem Senses. 2015;40(7):50716. https//doi.org/10.1093/chemse/bjv036

34. Running CA, Mattes RD. Different oral sensitivities to and sensations of short-, medium-and long-chain fatty acids in humans. Am J Physiol-Gastr L. 2014;307(3):G381-G389.

35. Harper. Bioquímica llustrada, 29. ${ }^{a}$ ed. Mc Graw Hill Lange; 2013, 792 pp.

36. Wade LG. Química Orgánica, 7. ed. México: Pearson Educación; 2012, 746 pp.

37. Wei W, Jin Q, Wang X. Human milk fat substitutes: Past achievements and current trends Progr Lipid Res. 2019;74:69-86. http://doi.org/10.1016/j.plipres.2019.02.001

38. Silencio JL, Lara G, Pérez Gil G, Montaño S, Ortiz RI, Castro MI, et. al. Ácidos grasos en el calostro y en la leche madura de mujeres mexicanas. Rev Mex Pediatr. 2012;79(1)5-11. Disponible en: https://www.medigraphic.com/pdfs/pediat/sp-2012/sp121b.pdf

39. Barreiro R, Díaz-Bao M, Cepeda A, Regal P. Fente Fatty acid composition of breast milk in Galicia (NW Spain): A cross-country comparison. Prostaglandins, Leukotrienes and Essential Fatty Acids. Prostaglandins Leukot Essent Fatty Acids 2018;135:102-14. http://doi.org/10.1016/j.plefa.2018.06.002

40. U.S. Department of Health and Human Services and U.S. Department of Agriculture. 2015-2020. Dietary Guidelines for Americans. $8^{\text {th }}$ ed. December 2015. Disponible en: https://health.gov/dietaryguidelines/2015/guidelines/

41. Belc N, Smeu I, Macri A, Vallauri D, Flynn K. Reformulating foods to meet current scientific knowledge about salt, sugar and fats. Trends in Food Sci Tech. 2019; 84:25-8. http://doi.org/10.1016/.tifs.2018.11.002

42. O'Sullivan MG. Nutritionally Optimised Low Fat Foods. En: A Handbook for Sensory and Consumer-Driven New Product Development. Innovative Technologies for the Food and Beverage Industry. Woodhead Publishing Series in Food Science; 2017, pp.177-96. http://doi.org/10.1016/B978-0-08-100352-7.00009-9

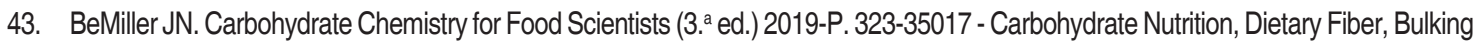
Agents, and Fat Mimetics. http://doi.org/10.1016/B978-0-12-812069-9.00017-0

44. Razavi SM, Behrouzian F. Biopolymers for Food Design Handbook of Food Bioengineering. En: Grumezescu AM, Holban AM (eds.). Handbook of Food Bioengineering. Academic Press; 2018. pp. 65-94. http://doi.org/10.1016/B978-0-12-811449-0.00003-7

45. Ma Y, Cai CH, Wang J, WenSun D. Enzymatic hydrolysis of corn starch for producing fat mimetics. J Food Eng. 2006;73:297-303. http://doi.org/10.1016/j.jfoodeng.2005.01.023

46. Chen $Y$, She $Y$, Zhang $R$, Wang J, Zhang X, Gou X. Use of starch-based fat replacers in foods as a strategy to reduce dietary intake of fat and risk of metabolic diseases. Food Sci Nutr. 2020; 8: 16-20. http://doi.org/10.1002/fsn3.1303

47. Gibis $M$, Schuh V, Weiss J. Effects of carboxymethyl cellulose (CMC) and microcrystalline cellulose (MCC) as fat replacers on the microstructure and sensory characteristics of fried beef patties. Food Hydrocolloid. 2015;45:236-46. http://doi.org/10.1016/j. foodhyd.2014.11.021 


\section{El gusto a grasa}

48. Ahmadi P, Tabibiazar M, Roufegarinejad L, Babazadehd A. Development of behenic acid-ethyl cellulose oleogel stabilized pickering emulsions as low calorie fat replacer. Int J Biol Macromol. 2020;150;974-81. http://doi.org/10.1016/.j.jbiomac.2019.10.205

49. Zheng Y, Zheng M, Ma Z, Xin B, Guo R, Xu X. Sugar Fatty Acid Esters. En: Ahmad M, Xu X (eds.). Polar lipids biology, chemistry, and technology. Academic Press and AOCS Press; 2015; pp. 215-43. http://doi.org/10.1016/B978-1-63067-044-3.50012-1

50. Chung C, Degner B, McClements DJ. Development of Reduced-calorie foods: Microparticulated whey proteins as fat mimetics in semi-solid food emulsions. Food Res Int. 2014;56:136-45. http://doi.org/10.1016/.foodres.2013.11.034

51. Liu R, Wang L, Liu Y, WuT, Zhang M. Fabricating soy protein hydrolysate/xanthan gum as fat replacer in ice cream by combined enzymatic and heat-shearing treatment. Food Hydrocolloid. 2018;81:39-47. http://doi.org/10.1016/j.foodhyd.2018.01.031

52. Johnson ME. Cheese: Low- fat and reduced fat-cheese. In reference module in Food Science. En: Encyclopedia of Dairy Sciences; 2011, pp. 833-42. http://doi.org/10.1016/B978-0-08-100596-5.00672-7

53. Jalal H, Para PA, Ganguly S, Padhy A, Praveen PK, Wakchaure R. Fat replacers in meat: a brief review. Word Journal of Enineering Research and Technology. 2015;1:(2)16-21. Disponible en: https: // www.wjert.org/archive show/2015/11/NOLUME-1-OCTOBERISSUE-2

54. Riosa R, Garzón R, Lannes SC, CM Rosella. Use of succinyl chitosan as fat replacer on cake formulations. LWT - Food Sci Tech. 2018;96:260-5. http://doi.org/10.1016/j.lwt.2018.05.041

55. ADA. Position of the American Dietetic Association: Fat Replacers. J Am Diet Assoc. 2005;105:266-75. http://doi.org/10.1016/j. jada.2004.12.011

56. McClements DJ. Reduced-Fat Foods: The Complex Science of Developing Diet-Based Strategies for Tackling Overweight and Obesity. American Society for Nutrition. Adv. Nutr. 2015. 6: 338S-52S. http://doi.org/10.3945/an.114.006999 